\title{
PROBABILISTIC ASSESSMENT OF PIPELINE - FAULT CROSSING
}

\author{
Vasileios E. Melissianos ${ }^{1}$, Dimitrios Vamvatsikos ${ }^{2}$, and Charis J. Gantes ${ }^{3}$ \\ ${ }^{1} \mathrm{JB}$ ATE \\ 30, Itanou Str., GR-71307 Herakleion, Crete \\ e-mail: melissianosv@gmail.com \\ ${ }^{2}$ National Technical University of Athens \\ 9, Iroon Polytechneiou Str., GR-15780 Zografou Campus, Athens \\ divamva@central.ntua.gr \\ ${ }^{3}$ National Technical University of Athens \\ 9, Iroon Polytechneiou Str., GR-15780 Zografou Campus, Athens \\ chgantes@central.ntua.gr
}

Keywords: buried steel pipeline, fault crossing, fault displacement, seismic risk assessment, probabilistic approach.

\begin{abstract}
Buried steel pipelines transporting oil and oil products play a vital role in the energy supply chain. Pipelines extend to long distances and thus intercepting tectonic faults, when a seismic area is crossed, is often inevitable and may heavily threat the pipeline integrity. Earthquakes and the associated fault displacements are naturally random events and therefore the imposed large ground displacements on the pipeline have to be considered through a probabilistic perspective. In the present study, a comprehensive seismic risk analysis of buried pipeline - fault crossing is presented, consisting of two steps. The first step is the probabilistic assessment of the fault displacement accounting also for the pertinent uncertainties. The second step is the pipeline structural analysis. The transition from the seismological data to the structural analysis is realized through the fault displacement components as the selected vector intensity measure. The outcome of the proposed methodology is the strain hazard curves for both tensile and compressive longitudinal strains. The resulting strain capacities are compared to strain demands from structural codes in order to assess the potential of pipeline failure due to local buckling or tensile fracture. Furthermore, uncertainty and disaggregation results from the fault displacement hazard analysis are presented for the selection of the appropriate deterministic design scenario and the evaluation of the fault displacement hazard parameters. Lastly, the proposed process is a reliable estimation tool for seismic risk assessment of pipeline - fault crossing and a decision making tool for route selection and application of preventive measures against the consequences of faulting on pipelines.
\end{abstract}




\section{INTRODUCTION}

Industrial experience has demonstrated over the years that the most efficient way for transporting hydrocarbonates over long distances is buried steel pipelines with continuous welded joints. Pipelines are structures of high importance acknowledging that any potential failure may have devastating consequences on the environment, nearby residential areas, as well as economic losses. Thus, pipelines are designed and constructed within a stringent framework of standards and regulations as societies are highly concerned about such projects. So, the combination of the route selection restrictions and pipelines' extended length between oil producers and oil consumers leads several times to the transverse of seismic areas that usually incorporate areas prone to large ground displacements, such as tectonic faults. Fault activation due to earth plate movement imposes large ground displacements that the pipeline has to accommodate safely. Investigation of previous earthquake events has demonstrated that the premier cause of pipeline failure is the imposed large differential ground displacement due to fault offset, compared to other seismic induced actions, such as seismic wave propagation, liquefaction induced lateral spread or landslide [1]. Possible failure modes in such situations are wall local buckling/wrinkling due to compressive strains, tensile fracture of girth welds due to tensile strains and in case of reverse fault type, upheaval buckling due to compressive forces.

Hydrocarbonate pipelines are classified as hazardous structures given the consequences of a potential failure. Thus, it is deemed appropriate to perform a comprehensive risk analysis of pipeline - fault crossing hazard by incorporating the uncertainty of the loading, i.e. fault offset, and the response of the pipeline - soil interaction system. Even though fault rupture is a rare event, most pipeline operators are willing to accept the damage related to fault activation, as long as the pipeline's integrity is maintained, i.e. no leak or rupture. However, earthquakes and the associated fault displacements are naturally random events and thus the dominant question during the design pertains to the appropriate magnitude of fault offset that has to be taken into account. Additionally, the seismic risk analysis is useful in order to design or evaluate financial (insurance) or physical mitigating measures (retrofitting or upgrading) against the fallouts of fault offset. Regarding the construction of a new pipeline project, hazard estimation is necessary so that the appropriate measures are taken to control the subsequent levels of risk through redirecting the design procedure. With reference to the financial loss estimation for insurance purposes, the quantification of failure hazard is the vital information required to fix premiums [2].

The proposed procedure for seismic risk analysis of buried pipeline - fault crossing consists of two parts: (i) conduct the probabilistic analysis of fault displacement hazard, (ii) perform pipeline structural analysis and then merge the results to conduct the seismic risk analysis. The basic aspects of the theoretical background of the proposed procedure for pipeline - fault crossing have been presented in previous work of the authors [3]. The appropriate tool for the probabilistic analysis of the fault displacement hazard is the Probabilistic Fault Displacement Hazard Analysis (PFDHA) introduced by Youngs et al. [4], which aims at quantifying the mean annual rate of exceeding various fault displacement levels at the site of pipeline - fault crossing. PFDHA integrates available geological and seismological data including, for example, the probability of fault activation, rupture location, fault slip rate, distribution of earthquake magnitudes and their associated uncertainties. Then, the seismic risk analysis is the combination of the results for developing the hazard curves for the selected intensity measure and the quantification of the potential of pipeline failure. 


\section{SEISMIC HAZARD ASSESSMENT}

\subsection{Probabilistic vs. determinist approach}

In seismic engineering there are two approaches for seismic risk assessment: the first one is the deterministic that is implemented through the Deterministic Seismic Hazard Analysis (DSHA) and the second is the probabilistic one applied trough the Probabilistic Seismic Hazard Analysis (PSHA).

The aim of DSHA is the development of earthquake scenarios that consist of a postulated occurrence of an earthquake of a specified magnitude, namely the characteristic or critical earthquake at a specific location, which could affect the structure under consideration. The DSHA implementation does not provide any information regarding the expected level of shaking or fault displacement during the structure's life time; it does not account for the earthquake occurrence probability or the effect of uncertainties throughout the various methodology steps. In proportion to DSHA that deals with ground shaking (e.g. PGA estimation), the Deterministic Fault Displacement Hazard Analysis (DFDHA) has been developed to deal with fault offset hazard.

PSHA, then, aims at quantifying the probabilistic nature of earthquake effects. The hazard derived from different seismic sources is considered separately and the influence of every source on the final outcome is transparent [2]. On the other hand, the contribution of all sources under consideration is combined through PSHA into a single exceedance rate for the specified ground-motion parameter (e.g. PGA). This leads to the estimation of a range of parameters, such as the spectral ordinates at several periods; thus the results may not be compatible with any physically feasible earthquake scenario. The pipeline - fault crossing derives special attention both during the design and the life-cycle of a pipeline project, as the consequences of a potential pipeline failure are usually grave. Thus, the probabilistic approach is selected for the seismic risk assessment of pipeline - fault crossing assisted by our generally incomplete knowledge about the variable nature of earthquake loading and the complex properties of the coupled pipeline - soil system. Within the probabilistic framework the most suitable tool for seismic risk assessment of buried pipeline - fault crossing is the Probabilistic Fault Displacement Hazard Analysis (PFDHA) that quantifies the probabilistic nature of the loading. The basis of PFDHA was presented in [5] where the required data for assessing the probability of rupture for a given fault and its effect on a site under investigation were presented. Then, Youngs et al. [4] presented the framework of PFDHA in order to quantify the mean annual rate of exceeding various fault displacement levels at a site by considering the fault location, the fault slip rate, the strongest expected earthquake, as well as their epistemic uncertainties.

\subsection{Pipeline - fault crossing geometry}

A straight pipeline segment without any bends is considered that intercepts a single fault at a specific location and thus the crossing site is represented by a single point. The fault is assumed to be planar appearing on the ground surface as a straight line and the fault thickness is approximated to zero. The pipeline - fault crossing is schematically illustrated in Figure 1, therein $\beta$ is the pipeline - fault crossing angle, $L F$ the fault length, $L_{p}$ the crossing site from the fault closest edge and $\psi$ the fault dip angle. The fault displacement is defined by $D$ and is related to the spatial components $\left[\Delta_{1}, \Delta_{2}, \Delta_{3}\right]$, where $\Delta_{1}$ and $\Delta_{2}$ are the fault parallel and fault perpendicular horizontal components, while $\Delta_{3}$ represents the vertical component:

$$
D=\sqrt{\Delta_{1}^{2}+\Delta_{2}^{2}+\Delta_{3}^{2}}
$$




$$
\Delta_{3}=\Delta_{2} \tan \psi
$$

When rotating the fault coordinate system $(1,2,3)$ horizontally by the angle $\beta$, then $\Delta_{1}$ and $\Delta_{2}$ are transformed to $\Delta_{\mathrm{x}}$ and $\Delta_{\mathrm{y}}$, i.e. the longitudinal and the transverse displacements with respect to the pipeline axis, while the vertical displacements $\Delta_{3}$ and $\Delta_{\mathrm{z}}$ coincide:

$$
\begin{aligned}
& \Delta_{x}=\Delta_{1} \cos \beta+\frac{\Delta_{3}}{\tan \psi} \sin \beta \\
& \Delta_{y}=-\Delta_{1} \sin \beta+\frac{\Delta_{3}}{\tan \psi} \cos \beta
\end{aligned}
$$

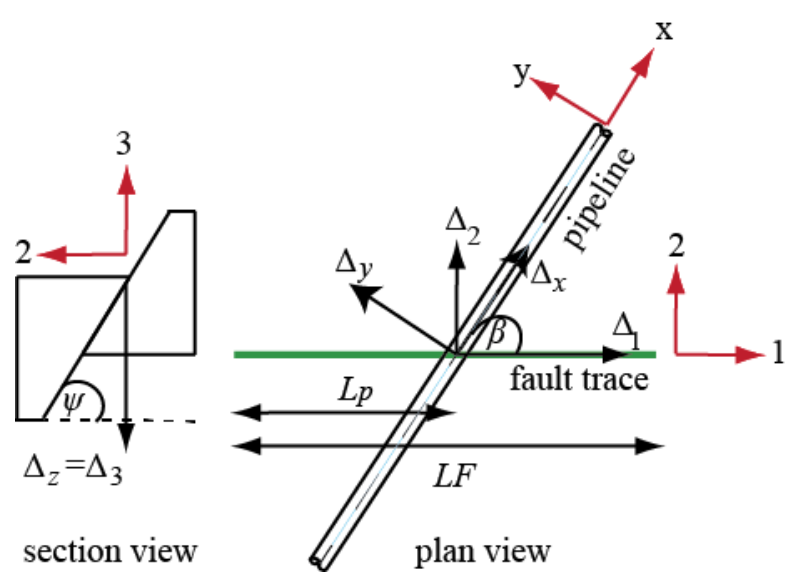

Figure 1: Pipeline fault crossing plan and section view.

\subsection{Probabilistic fault displacement hazard analysis}

The PFDHA methodology includes two discrete approaches within it: the "earthquake approach" and the "displacement approach". The "earthquake approach" is directly derived from the PSHA, as developed by Cornell [6], and explicitly relates the occurrence of fault displacement on a fault at a site near or at the ground surface to the occurrences of earthquakes in the site region. On the other, the "displacement approach" needs extensive recorded or paleoseismic data. However, these data are not always available and thus within the proposed methodology, the "earthquake approach" is adopted. Furthermore, in the "earthquake approach" of PFDHA the distinction among two types of faulting is introduced, namely the "principal faulting" and the "distributed faulting". The former type is adopted hereinafter, assuming that pipeline crosses a single and sufficiently recorded fault that its displacement may be represented along a single narrow trace or over a zone that be may a few meters wide.

The required information to assess any potential consequences of faulting on pipeline integrity is provided by PFDHA. This information assists engineers on risk-informed decisions regarding site suitability when geological investigations are inconclusive. The outcome, then, of PFDHA is the fault displacement hazard curve on pipeline crossing site, where the fault displacement is presented versus the mean annual rate of exceeding every fault displacement values:

$$
v_{k}(d)=\sum_{n} \alpha_{n}\left(m^{0}\right) \times \int_{m^{0}}^{m_{n}^{u}} f_{n}(m) \times\left[\int_{0}^{\infty} f_{k n}(r \mid m) \times P_{k n}^{*}(D>d \mid m, r) d r\right] d m
$$

where $\alpha_{n}\left(m^{0}\right)$ is the rate of all earthquakes on source $n$ above a minimum magnitude of engineering significance $m^{0}, f_{n}(m)$ is the probability density of earthquake size that seismic source 
$n$ can produce between values $m^{0}$ and $m_{n}{ }^{u}$ estimated using the Gutenberg - Richter Bounded Recurrence Law [7], $f_{k n}(r \mid m)$ is the conditional probability density function for distance from site $k$ to an earthquake of magnitude $m$ occurring at source $n$. Finally, $P^{*}(D>d \mid m, r)$ is the conditional probability that, given an earthquake of magnitude $m$ at distance $r$ from crossing site, the fault displacement will exceed displacement $d$. In practice it relates the occurrence of fault displacement at the crossing site to the occurrence of an earthquake through an attenuation model and thus is referred as the Displacement Attenuation (or prediction) Function, which differs from the usual ground motion prediction. Moreover, this term is the product of the conditional probability of slip and the conditional probability of exceedance.

\section{PROPOSED METHODOLOGY}

\subsection{Conceptual algorithm}

The estimation of fault displacement hazard in terms of practical applications depends on three factors: (i) the earthquake magnitude as the key factor for describing a seismic source, which ranges from a minimum value $M_{\min }$ to a maximum value $M_{\max }$ and is discretized into a numbers of bin to account for all possible values, (ii) the surface rupture length (SRL) along the fault trace, acknowledging that different earthquakes may rupture lengths of different size and (iii) the SRL position on the fault trace, as the location of a given earthquake may not encounter the pipeline - fault crossing.

The uncertainty of fault rupture intercepting the pipeline crossing site is handled by considering a variety of potential SRLs, each at a different location. Without more detailed data being available, SRLs of the same size are considered to be equiprobable. Additionally, for simplicity of bookkeeping, a minimum SRL size is determined as for example corresponding to the minimum magnitude of interest via empirical Equations and all subsequent larger sizes are simply integer multiples. So, every SRL size is accounted for at all possible positions, keeping track of those where the rupture crosses the pipeline and thus contributing to the pipeline displacement hazard.

The PFDHA, as presented in section 2.3, is employed as an application of the total probability theorem trough a conceptual algorithm that deals with only one seismic source, i.e. $n=1$. The mean annual rate of exceeding a defined fault displacement value $\lambda_{D}(d)$ at the pipeline crossing site is estimated as:

$$
\lambda_{D}(d)=v_{o} \times \sum_{i} P\left(D>d \mid m_{i}\right) \times f_{M}\left(m_{i}\right)
$$

where $v_{o}$ stands for the rate of all earthquakes above a minimum magnitude of engineering significance and is calculated based either on the Equation describing the seismicity of the investigated seismic source zone or provided by the engineer as an input parameter and $f_{M}\left(m_{\mathrm{i}}\right)$ is the earthquake probability calculated according to Gutenberg-Richter Bounded Recurrence Law [7]. Probability function $P\left(D>d \mid m_{i}\right)$ estimates the probability that fault displacement exceeds a defined value $d$ given earthquake magnitude $m_{\mathrm{i}}$ and is summed over all magnitude values as centers of bins. Further breakdown of Equation (5) yields to:

$$
P\left(D>d \mid m_{i}\right)=\sum_{i} \sum_{j} \sum_{k} P\left(D>d \mid m_{i}, S R L_{j}, F D_{k}, P_{l}\right) \times P\left(S R L_{j}, F D_{k}, P o s_{l} \mid m_{i}\right)
$$

In order to calculate the probability function $P\left(D>d \mid m_{\mathrm{i}}\right)$ of Equation (5), apart from earthquake magnitude range discretization in $i$ bins and rupture length discretization in $j$ bins, a third discretization for the fault displacement option $(F D)$ of PFDHA is necessary. It is pointed out that the proposed conceptual algorithm applies equally for the maximum fault dis- 
placement (MD) and the average fault displacement (AD) option of PFDHA. Then, Equation (6) has two constituent elements, the first term is the conditional probability of exceedance and the second term is the conditional probability of SRL, rupture position along the fault trace and the FD approach of PFDHA given an earthquake magnitude.

The conditional probability of exceedance (6) is the term $P\left(D>d \mid m_{i}, S R L_{j}, F D_{k}, P o s_{l}\right)$ that stands as the core of the PFDHA and necessitates detailed calculations that are carried out over each combination of bins of earthquake magnitude, rupture length, fault displacement and all possible positions of $S R L_{j}$ along the fault trace. The second element of Equation (6) is the conditional probability of SRL, rupture position along the fault trace and the $F D$ approach of PFDHA given an earthquake magnitude $m_{i}$, which is decomposed in:

$$
P\left(S R L_{j}, F D_{k}, \operatorname{Pos}_{l} \mid m_{i}\right)=P\left(S R L_{j}, F D_{k} \mid m_{i}\right) \times P\left(\operatorname{Pos}_{l} \mid m_{i}\right)=P\left(S R L_{j}, F D_{k} \mid m_{i}\right) \times \frac{1}{N_{l}}
$$

where the first term is the probability of a given range of fault displacement and size of SRL to occur given an earthquake magnitude. However, $S R L_{j}$ and $F D_{k}$ are well correlated given the earthquake magnitude. Therefore, for each combination $(i, j, k)$ the value of $P\left(S R L_{j}, F D_{k} \mid m_{i}\right)$ needs to be estimated with the appropriate joint distribution $f\left(S R L_{j}, F D_{k} \mid m_{i}\right)$. The best known such distribution comes from Wells and Coppersmith [8] and allows the definition of $f\left(S R L_{j}, F D_{k} \mid m_{i}\right)$ as a joint lognormal with positive correlation. Assuming that the discretization is sufficiently fine, the probability of $S R L$ and $F D$ falling in a bin given the magnitude can be approximated via a single PDF value at its center:

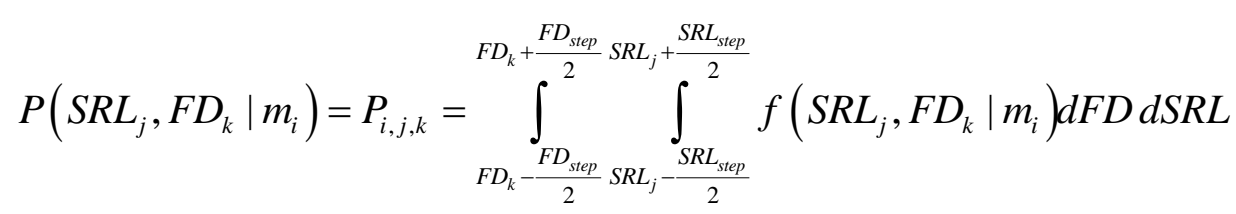

$$
\begin{aligned}
& \cong f\left(S R L_{j}, F D_{k} \mid m_{i}\right) \times \Delta F D \times \Delta S R L
\end{aligned}
$$

Still, the sum of $P_{i, j, k}$ over $j$ and $k$ for all $S R L_{j}$ and $F D_{k}$ should equal one. Thus, the required probability function is renormalized as:

$$
P\left(S R L_{j}, F D_{k} \mid m_{i}\right)=\frac{P_{i, j, k}}{\sum_{j} \sum_{k} P_{i, j, k}}
$$

\subsection{Interface variable}

In seismic risk analysis the roles of the seismologist and the structural engineer are linked by the use of an interface variable, known as the intensity measure (IM). In pipeline - fault crossing seismic risk analysis the structural engineer needs to estimate the mechanical behavior of the pipeline to given IM values. The proper such values are the fault displacement spatial components $\Delta_{1}, \Delta_{2}$ and $\Delta_{3}$, as defined in section 2.2. The spatial distribution of fault components is not only necessary for the fault characterization as normal, strike-slip or reverse, but also dominates the pipeline's response. The latter is of great importance as normal faulting leads to pipeline bending and tension, strike-slip faulting leads to pipeline bending and tension or compression, while reverse fault leads to pipeline bending and compression. Thus, it is deemed necessary to define a vector intensity measure [9]. The appropriate such vector IM consists of two components, namely $\left[\Delta_{1}, \Delta_{3}\right]$, which are structure independent and can fully describe the loading of the structural model, given that $\Delta_{2}$ component is functionally 
dependent to them. Moreover, the selected IM covers the design goal of assessing the structural behavior of the pipeline due to faulting. However, if the proposed methodology needs to be extended to encompass frequency related events, such as low-cycle fatigue, then the adopted IM might be insufficient.

\subsection{Fault displacement components}

The outlined PFDHA methodology deals with the magnitude of fault displacement norm, neglects its spatial orientation and passes up the fact that fault displacement in nature is usually three-dimensional. Thus, the necessity arises to introduce a calculation procedure of components' magnitude with reference to fault type. So, fault types are divided into two categories, namely strike-slip type where offset is dominated by $\Delta_{1}$ component and normal or reverse type where the $\Delta_{3}$ component is dominant. However, there are not sufficient published data regarding the spatial distribution of the components. Hence, within the scope of the proposed methodology, the dominant component is calculated assuming it is normally or uniformly distributed. Due to the lack of adequate data, the distributions adopted are reasonable assumptions and shall be adjusted or replaced if reliable data are available in the future.

In case the dominant fault component is assumed to be uniformly distributed, it is estimated as:

$$
\Delta_{1} \text { or } \Delta_{3}=(0.70 D \div 0.90 D)
$$

where the discretization, the lower and the upper bound are input engineering parameters. The proposed lower and upper bounds stand for a reasonable estimation to indicate the dominance of the component that characterizes the fault type and at the same time to assure that fault offset remains three-dimensional.

In case the dominant fault component is assumed to be normally distributed, it is estimated using the parameters listed in Table 1.

\begin{tabular}{ll}
\hline Parameter & Value \\
\hline$\mu$ & $0.80 D$ \\
$\sigma$ & $0.20 \times \mu$ \\
Upper bound & $0.90 D$ \\
Lower bound & $0.70 D$ \\
\hline
\end{tabular}

Table 1: Normal distribution parameters for dominant fault component calculation.

The component's mean annual rate of density, whether $\Delta_{1}$ is the dominant component for strike-slip fault, or $\Delta_{3}$ for normal/reverse fault, respectively, is estimated as:

$$
\begin{aligned}
& \lambda^{\prime}\left(\Delta_{1}, \Delta_{3}\right)=f\left(\Delta_{1} \mid D\right) \times \frac{d \lambda_{D}}{d D} \times \frac{\partial \Delta_{3}}{\partial D} \\
& \lambda^{\prime}\left(\Delta_{3}, \Delta_{1}\right)=f\left(\Delta_{3} \mid D\right) \times \frac{d \lambda_{D}}{d D} \times \frac{\partial \Delta_{1}}{\partial D}
\end{aligned}
$$

where $f\left(\Delta_{1} \mid D\right)$ is the distribution probability density function (either normal or uniform) of the dominant component (either $\Delta_{1}$ or $\Delta_{3}$ ), $\mathrm{d} \lambda_{D} / \mathrm{d} D$ is the rate density for given $D$, while $\partial \Delta_{1} / \partial D$ or $\partial \Delta_{3} / \partial D$ is the derivative of the dominant fault component over $D$ that is necessary in order to change the variable [10]. It should be noted that $\lambda^{\prime}\left(\Delta_{1}, \Delta_{3}\right)$ is not rate, but rate density and thus 
an integration of $\lambda^{\prime}\left(\Delta_{1}, \Delta_{3}\right)$ over a two-dimensional interval of $\Delta_{1}$ and $\Delta_{3}$ yields the mean annual rate of events occurring in this interval.

\subsection{Pipeline strain hazard curves}

Fault offset is the primary cause of pipeline failure due to earthquake events, as the differential ground movement imposes large permanent ground displacements on the buried pipeline that the pipeline has to accommodate. Thus, high levels of tensile and compressive strains are developed and endanger the pipeline's integrity. Compressive strains may lead to local buckling of the wall, while tensile strains may lead to fracture of the girth welds between adjacent steel parts. So, the primary consideration for pipeline earthquake resistant design is the determination of strain capacity and for this reason, strain hazard curves are the most suitable tool to perform a probabilistic estimation of any potential pipeline failure throughout the pipeline life-time. However, whilst structural analyses provide strain demands, strain capacity terms are adopted by code recommendations. ALA [11] provisions suggest for the longitudinal strains resulting from ground movement due to fault offset the tensile strain limit $\varepsilon_{t, c}=2 \%$ and the compressive limit $\varepsilon_{c, c}$ of:

$$
\begin{aligned}
\varepsilon_{c, c} & =0.5\left(\frac{t}{D^{\prime}}\right)-0.0025+3000\left(\frac{p D_{e}}{2 E t}\right)^{2} \\
& \text { where } D^{\prime}=D_{e} /\left[1-3\left(D_{e}-D_{\text {min }}\right) / D_{e}\right]
\end{aligned}
$$

where $t$ is the pipeline wall thickness, $D_{e}$ is the pipeline external diameter, $D_{\min }$ is the internal diameter, $p$ is the internal pressure and $E$ the pipeline steel modulus of elasticity. Equation (13) includes a term for internal pressure, which acts as a relief against the external earth pressure. In the present study the internal pressure is assumed to be equal to zero, as a less favorable situation, thus the pertinent term is neglected.

Strain hazard curves present the mean annual rate of exceeding a defined strain value $\lambda_{E}(\varepsilon)$ and are evaluated as:

$$
\lambda_{E}(\varepsilon)=\sum_{\varepsilon\left(\delta_{1 i}, \delta_{3 j}\right) \geq \varepsilon} \Delta \lambda\left(\delta_{1 i}, \delta_{3 j}\right)
$$

where $\Delta \lambda\left(\delta_{1 i}, \delta_{3 j}\right)$ is the mean annual rate of equaling or reaching a given range of fault displacement with components $\delta_{1 i}$ and $\delta_{3 j}$. It is estimated by integrating the mean annual rate of density $\lambda^{\prime}\left(\Delta_{1}, \Delta_{3}\right)$ in the appropriate range of components displacement $\delta_{1 i}$ and $\delta_{3 j}$. Finally, this result is in turn summed over all $i$ and $j$ values of $\Delta_{1}$ and $\Delta_{3}$ respectively that are found in the structural analysis to correspond to strain values larger than $\varepsilon$.

\subsection{Uncertainty}

PFDHA, from its origins, can incorporate any quantifiable uncertainty, as identification and quantification of uncertainties is integral to the seismic hazard analysis. Uncertainties are usually divided into two main categories, namely aleatory and epistemic. The division of uncertainties demands a distinct treatment and it is good practice to treat them separately, in order to identify the key uncertainties, although this is not always an easy task. The aleatory uncertainties refer to the inherent variability of the nature over time, while epistemic uncertainties are related to the inadequate understanding of the nature and can be in time reduced with better observations. In the present study, epistemic uncertainties are handled though a logic tree, as the latter is considered to be the state-of-the art tool to quantify and incorporate epistemic uncertainties [12]. The set-up of a logic tree includes the production of alternative 
models for various input variables and then the assignment of weight factors to tree branches. These factors represent the engineer's degree of belief in the alternative models or in other words they represent the degree of belief in one model with respect to another. Epistemic uncertainties are considered in the present study through the logic tree that is presented in Figure 2 and includes three parameters.

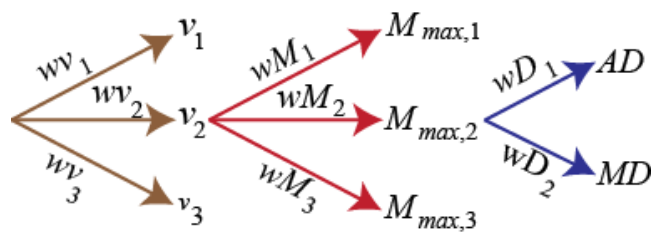

Figure 2: Uncertainty analysis logic tree.

The first parameter is the seismic rate $v$ as the main characteristic of the seismic source that is under question, given that seismologists usually provide the mean value. The second is the maximum expected earthquake magnitude $M_{\max }$. While the minimum earthquake magnitude is selected based on the assumption that lower magnitudes do not contribute to seismic hazards of engineering significance, the maximum earthquake magnitude is uncertain. The third uncertainty parameter is the choice of the average fault displacement (AD) or the maximum earthquake displacement (MD) approach of PFDHA, as presented in section 3.1.

\section{ILLUSTRATIVE EXAMPLE}

The proposed methodology for seismic risk assessment of buried pipeline - fault crossings consists of two interrelated steps: (i) the seismological part, where the probabilistic fault displacement hazard analysis is conducted to extract the mean annual rate of exceeding fault displacement and the intensity measure on crossing site and (ii) the structural part, where the structural analysis of the buried pipeline is performed to extract the strain demands. Then, the results of both steps are combined, yielding the pipeline strain hazard curves. To demonstrate the proposed methodology a numerical example and its associated results are hereinafter presented.

\subsection{Example of fault displacement hazard analysis}

A normal fault is considered with length equal to $L F=100 \mathrm{~km}$, fault dip angle $\psi=70^{\circ}$ and average seismic rate $v=1.2$. Pipeline crossing is located $L_{p}=40 \mathrm{~km}$ from the fault closest edge, while pipeline - fault crossing angle equals $\beta=80^{\circ}$, with reference to Figure 1 . The minimum earthquake magnitude under consideration is selected as $M_{\min }=4.5$ and the average maximum earthquake magnitude is $M_{\max }=7.3$. Analysis is carried out for fault displacement values ranging from $0.01 \mathrm{~m}$ to $3 \mathrm{~m}$. Uncertainty parameters considered in the analysis are listed in Table 2 with respect to Figure 2. Regarding the AD or MD approach of PFDHA, they are both assigned a weight factor of 0.50 , given that there is not sufficient data to favor one approach compared to the other. For the same reasoning, both the normal and the uniform distribution approach for estimating the dominant fault component are assigned a weight factor of 0.50 . In addition, the component calculation procedure is an approximation and thus one cannot favor the uniform distribution against the normal distribution. 


\begin{tabular}{ll}
\hline Parameter value & Weight factor \\
\hline$v_{1}=1.1$ & $v_{1}=0.30$ \\
$v_{2}=1.2$ & $v_{1}=0.40$ \\
$v_{3}=1.3$ & $v_{1}=0.30$ \\
$M_{\max , 1}=7.2$ & $v M_{1}=0.20$ \\
$M_{\max , 2}=7.3$ & $v M_{2}=0.60$ \\
$M_{\max , 3}=7.4$ & $v M_{3}=0.20$ \\
AD approach & $v A_{1}=0.50$ \\
MD approach & $v A_{2}=0.50$ \\
Normal distribution & $v F_{1}=0.50$ \\
Uniform distribution & $v F_{2}=0.50$ \\
\hline
\end{tabular}

Table 2: Uncertainty parameters considered in the illustrative example.

The output of PFDHA is the weighted average fault displacement hazard curve that is illustrated in Figure 3, where in logarithmic scale the mean annual rate or exceedance $\lambda_{D}(d)$ is presented on the horizontal axis, while the fault displacement on crossing site $d$ is presented on the vertical axis. The descending curve shape is predictable as the larger the fault displacement is, the lower the mean annual rate of exceedance is. The fault displacement components hazard surfaces are estimated according to section 3.3 and depicted in Figure 4, where on the vertical axis the mean annual rate of density is presented, while on the horizontal axes the two structural independent fault displacement components $\Delta_{1}$ and $\Delta_{3}$ are presented.

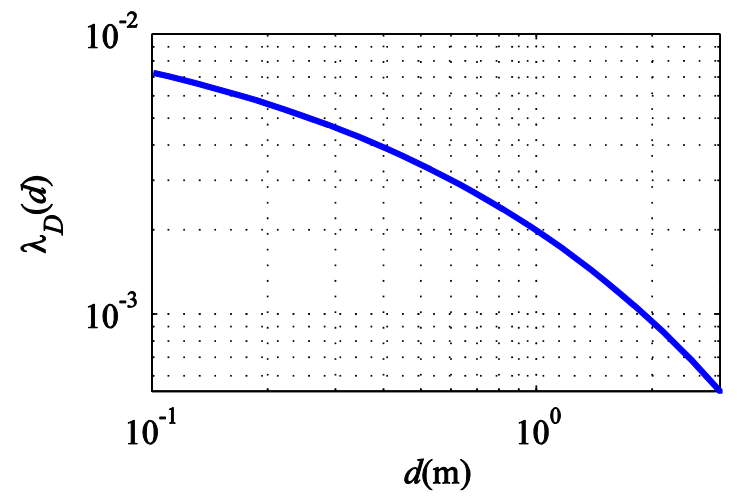

Figure 3: Weighted average fault displacement hazard curve on pipeline crossing site.

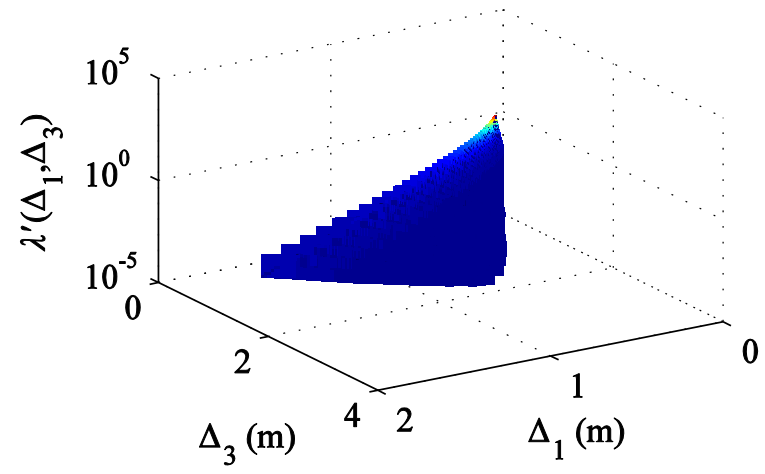

Figure 4: Weighted average fault components hazard surface on pipeline crossing site. 


\subsection{Disaggregation and uncertainty}

Uncertainties are an integral part of seismic risk assessment and are incorporated in the proposed methodology through the logic tree of Figure 2, where weight factors are assigned to tree branches. So, there are in total 18 "triplets" of $\left(w v_{i}, w M_{j}, w A_{k}\right)$ weight factors and in practice each branch corresponds to a single factor being the product of partial factors:

$$
w_{i, j, k}=w v_{i} \times w M_{j} \times w A_{k}
$$

Therefore, the weighted average hazard curve is produced as:

$$
\bar{\lambda}_{D}=\sum_{i, j, k} w_{i, j, k} \times \lambda_{D}^{i, j, k}(d)
$$

For the sake of simplicity, a Monte Carlo simulation approach can be adopted, assuming that the mean value $\mu$ and standard deviation $\sigma$ for a sample size of $N$ are evaluated as follows:

$$
\begin{aligned}
\mathrm{E}\left[\lambda_{D}\right]=\frac{\sum_{i=1}^{N} \lambda_{D}^{i}}{\mathrm{~N}} & =\frac{w_{1} \times \lambda_{D}^{1}+w_{2} \times \lambda_{D}^{2}+\ldots+w_{18} \times \lambda_{D}^{18}}{\mathrm{~N}} \\
\sigma & =\sqrt{\frac{1}{N} \times \sum_{i=1}^{N}\left(\bar{\lambda}_{D}-\lambda_{D}^{i}\right)^{2}}
\end{aligned}
$$

Then, the weighted average fault displacement hazard curve is illustrated in Figure 5 along with its dispersion, together with the mean minus one standard deviation.

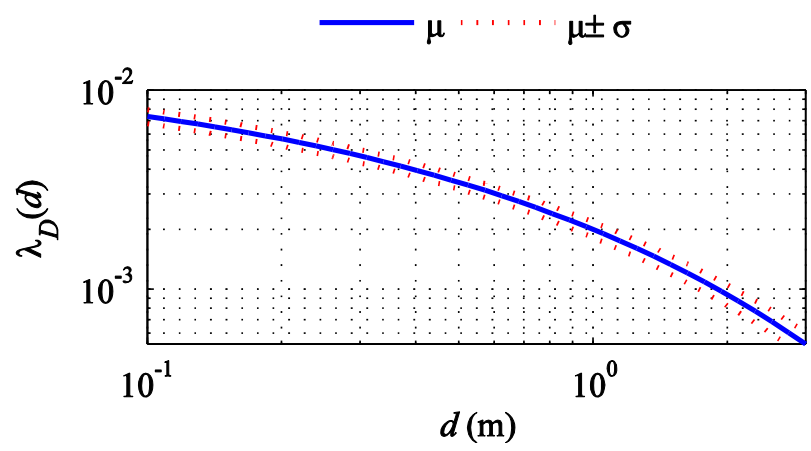

Figure 5: Weighted average fault displacement hazard curve with dispersion.

The conditional probability of exceedance that is incorporated in the fault displacement hazard calculation offers two approaches regarding the normalization of the displacement along the length of the rupture, i.e. the average fault displacement and the maximum fault displacement. As indicated previously, there are not adequate data to favor one approach against the other. Thus, disaggregation results are presented in order to evaluate the selection impact among the two approaches on the fault hazard curve. So, Figure 6 presents the weighted average fault displacement hazard curve adopting the AD or the MD approach of PFDHA, which indicates that for small displacement values the $\mathrm{AD}$ approach leads to higher values of mean annual rate of exceedance and vice versa for the MD approach. 


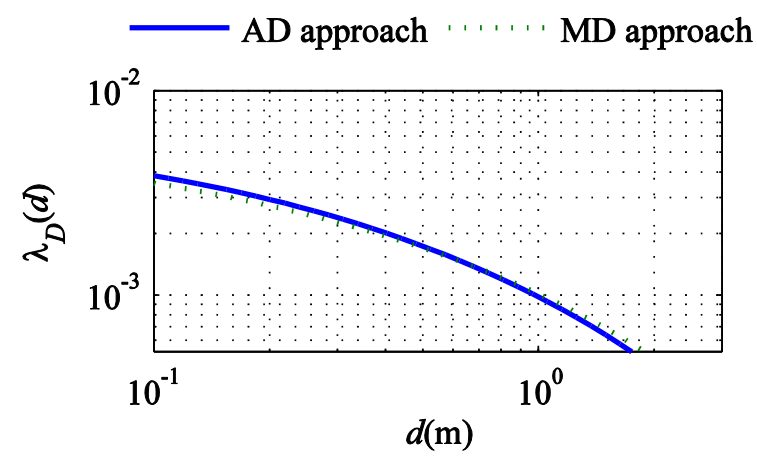

Figure 6: Weighted average fault displacement hazard curve adopting AD or MD approach of PFDHA.

In Eurocode 8 - Part 4 [13] two limit states are introduced for buried pipelines, namely the Ultimate Limit State (DLS) related to a return period of $T_{N C R}=475$ years or $10 \%$ probability of exceedance in 50 years and the Damage Limit State (DLS) related to a return period of $T_{D L R}=95$ years or $10 \%$ probability of exceedance in 10 years. The probability of exceedance is related with the mean annual rate of exceedance through the Equation (19).

$$
P=1-\exp \left(1-t / T_{\text {return }}\right) \Rightarrow P=1-\exp (-\lambda \times t) \Rightarrow \lambda=\frac{-\ln (1-P)}{t}
$$

Thus, the mean annual rate of exceeding the ULS is $\lambda_{U L S}=0.0021$ and the DLS is $\lambda_{D L S}=0.0105$. It is also useful to associate the limit states with earthquake magnitude in order to highlight the contribution of earthquake magnitude values, ranging from $M_{\min }=4.5$ to $M_{\max }=7.3$, to the limit states. Then, using the weighted average fault displacement hazard curve, by neglecting the uncertainty of the $M_{\max }$, it can be found through linear interpolation that the fault displacement corresponding to the ULS is $D_{U L S}=0.86 \mathrm{~m}$. For the DLS, there is no practically permanent fault offset for the fault under consideration for such low intensity and frequent earthquakes. The disaggregation on earthquake magnitude regarding the ULS is evaluated though:

$$
P_{m_{i}}=\frac{\lambda_{D}\left(D \mid m_{i}\right)}{\lambda_{D}(D)}
$$

The implementation of Equation (20) leads to Figure 7, where on the horizontal axis the earthquake magnitude values are plotted in bins and on the vertical axis their contribution to the limit states. Disaggregation results indicate that magnitude $m=7$ contributes most to the ULS. Larger magnitudes may cause larger displacements yet they are apparently too rare for this fault to figure more prominently for ULS. 


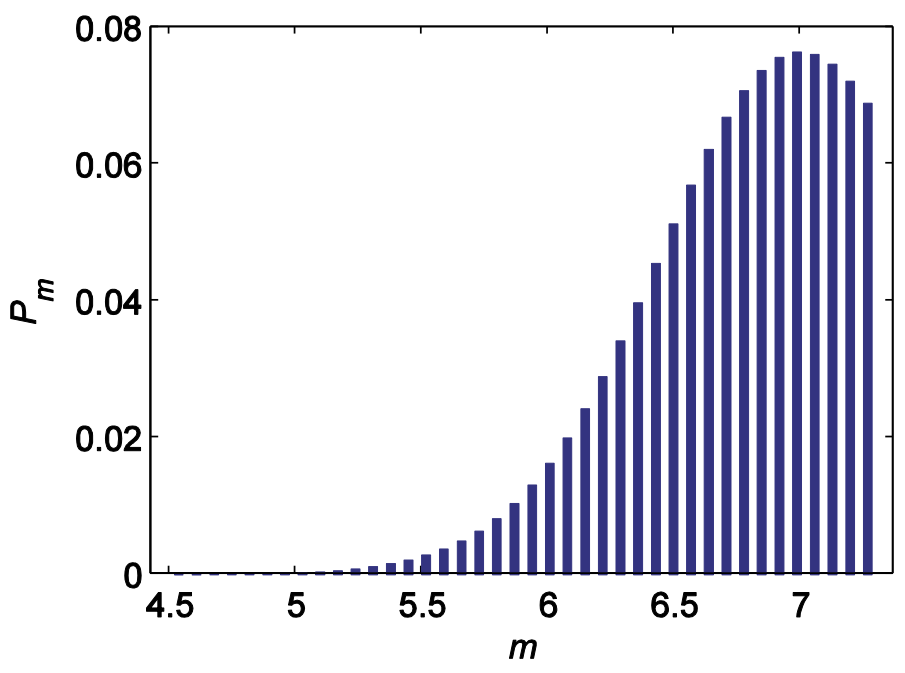

Figure 7: Disaggregation on earthquake magnitude with reference to Eurocode 8 pipeline ultimate limit state.

The spatial distribution of fault components is assessed in the present study through an approximate procedure. Due to the lack of sufficient data, the dominant component is calculated by adopting a uniform or a normal distribution. However, it is essential to demonstrate the differences among the two assumptions in terms of fault components hazard surfaces. Thereby, the independent $\Delta_{1}$ and $\Delta_{3}$ components are presented on the horizontal axes, while on the vertical axis their mean annual rate of density $\lambda^{\prime}\left(\Delta_{1}, \Delta_{3}\right)$ is presented. The hazard surface using the normal distribution is lower than the corresponding using the uniform distribution. It is also essential to recall that the mean annual rate of density is not a rate but a rate density and thus if an integration is carried out over a two-dimensional interval of $\Delta_{1}$ and $\Delta_{3}$ one can estimate the mean annual rate of occurrence in that interval.

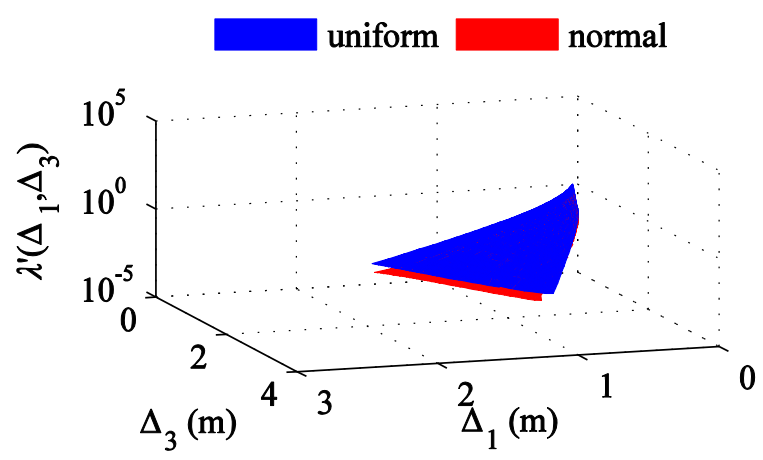

Figure 8: Fault displacement hazard surfaces adopting a uniform or a normal distribution.

\subsection{Pipeline structural analysis}

Pipeline structural analysis can be carried out either numerically or analytically in order to assess the pipe response due to faulting. The usual design process includes the use of analytical approaches in the preliminary design stage and then numerical simulations in the final stage. For the purposes of the illustrative example, the pipeline structural analysis is performed using the analytical procedure presented by Karamitros et al. in [14],[15] as a reliable approach to estimate the maximum and the minimum developing strain on the pipeline. 
A typical high-pressure natural gas API5L-X65 pipeline is considered, featuring an external diameter of $D_{e}=0.914 \mathrm{~m}$ (36in) and a wall thickness of $t=0.0127 \mathrm{~m}$. The pipeline steel is considered to be elastic with isotropic hardening, while its properties are listed in Table 3.

\begin{tabular}{ll}
\hline Parameter & Value \\
\hline Yield stress & $448.50 \mathrm{MPa}$ \\
Ultimate stress & $531.00 \mathrm{MPa}$ \\
Ultimate strain & $4.00 \%$ \\
Young's modulus & $210 \mathrm{GPa}$ \\
Yield strain & $0.233 \%$ \\
Hardening modulus & $1.088 \mathrm{GPa}$ \\
\hline
\end{tabular}

Table 3: API-5L X65 steel properties considered in the structural analyses.

The pipeline is assumed to be buried under $1.30 \mathrm{~m}$ of medium-density sand and coated with coal-tar. Backfill sand's friction angle is $\varphi=36^{\circ}$ and unit weight is equal to $\gamma=18 \mathrm{kN} / \mathrm{m}^{2}$. The surrounding soil is modeled as a series of mutually independent elastoplastic springs according to Winkler soil model, while their properties are estimated according to ALA [11] provisions and listed in Table 4.

\begin{tabular}{lll}
\hline Spring type & Force $(\mathrm{kN} / \mathrm{m})$ & Displacement $(\mathrm{m})$ \\
\hline Axial (frictional) springs & 22.7 & 3.0 \\
Transverse horizontal springs & 134.4 & 35.7 \\
Vertical upward springs & 41.2 & 2.6 \\
Vertical downward springs & 1021.8 & 95.3 \\
\hline
\end{tabular}

Table 4: Soil spring properties considered in the structural analyses.

\subsection{Pipeline strain hazard curves}

Within the scope of the present illustrative example, strain hazard curves are derived by combining structural analysis results and a basic determinist approach, where fault displacement hazard is represented by its weighted average (Figure 3), considering epistemic uncertainties. Also, strain capacities are considered to be deterministic, rather than uncertain, i.e. uncertainty regarding the pipeline steel properties and the soil properties and hence the pipeline mechanical behavior is neglected. The mean annual rate of exceeding a defined strain value is estimated via Equation (14). For the pipeline under investigation, the tensile strain limit equals $2 \%$, while the compressive strain limit yields $0.35 \%$. Then, the combination of analytical results with PFDHA results leads to the estimation of the pipeline strain hazard curves that are illustrated in Figure 9 and Figure 10 for the tensile and the compressive strains respectively. Strain hazard curves indicate that there is no risk of pipeline failure either due to tensile failure of girth welds, or due to local buckling. 


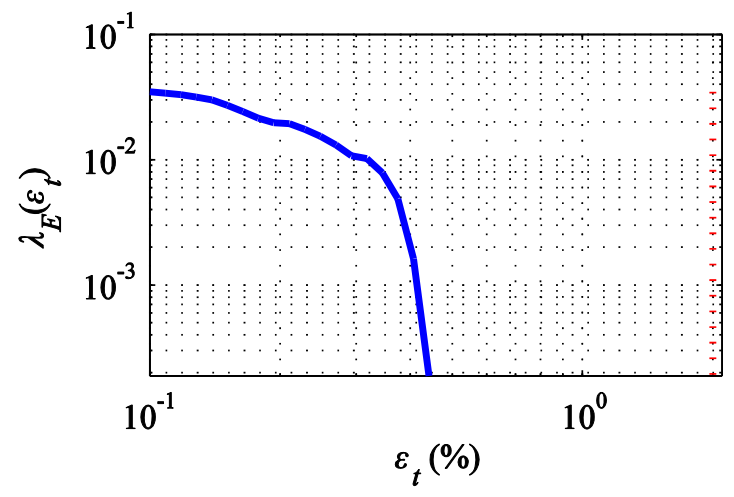

Figure 9: Tensile strain hazard curve.

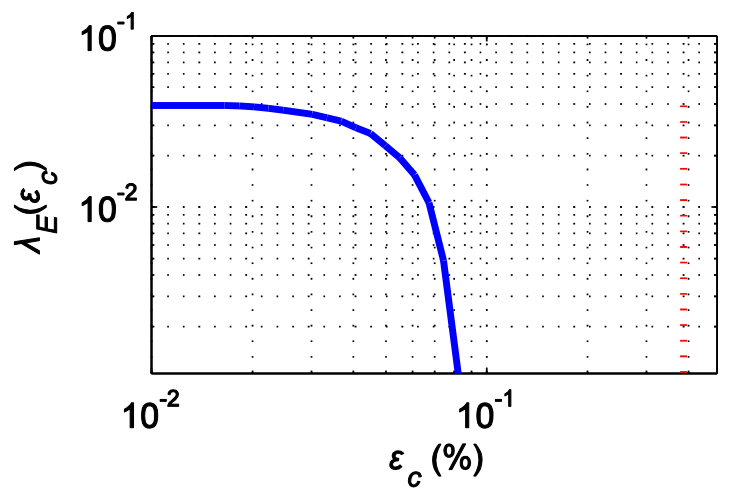

Figure 10: Compressive strain hazard curve.

\section{CONCLUSIONS AND REMARKS}

A two-step methodology for seismic risk assessment of buried steel pipelines crossing active faults is presented. The first step includes the Probabilistic Fault Displacement Hazard Analysis methodology as adjusted for pipeline fault crossing. The conceptual algorithm of the proposed methodology is also presented, by taking into account epistemic uncertainties. The second step is the "transition" from seismological data to structural analysis through the vector intensity measure of fault displacement components. Strain hazard curves are derived by associating results from pipeline mechanical behavior to hazard analysis in order to compute the mean annual rate of exceedance of longitudinal strains, which are compared to code-based strain failure criteria. Moreover, an illustrative example is presented to demonstrate the proposed methodology. Results regarding the uncertainty of adopting the average fault or the maximum fault displacement are presented to identify its impact on the mean annual rate of exceeding fault displacement on pipeline crossing site. Additionally, the fault components are estimated through an approximate calculation procedure and thus the differences between assuming a uniform or a normal distribution to estimate the dominant component are presented. Disaggregation results on earthquake magnitude with reference to Eurocode 8 limit states are displayed to evaluate the contribution of earthquake magnitude to the limit states.

The proposed approach offers a path for comprehensive performance-based assessment of buried pipelines crossing active faults. Using an adjusted PFDHA implementation for pipeline - fault crossing together with the pipeline response pipeline due to faulting using a reliable analytical approach, the hazard of pipeline crossing in terms of strains can be estimated. The proposed methodology provides engineers with a reliable estimation tool, admittedly requiring programing of the conceptual algorithm, but offering both seismic risk and pipeline structural analyses at low computational effort. 


\section{ACKNOWLEDGMENTS}

Financial support was provided by the European Research Executive Agency via Marie Curie grant PCIG09-GA-2011-293855 and by Greece and the European Social Funds through the Operational Program "Human Resources Development" of the National Strategic Framework (NSRF) 2007-2013.

\section{REFERENCES}

[1] M.J. O' Rourke, X. Liu, Response of buried pipelines subject to earthquake effects, Multidisciplinary Center for Earthquake Engineering Research. Buffalo, New York, 1999.

[2] J.J. Bommer, Deterministic vs. probabilistic seismic hazard assessment: an exaggerated and obstructive dichotomy. Journal of Earthquake Engineering, 6, Special Issue I, 4373, 2002.

[3] V.E. Melissianos, D. Vamvatsikos, C.J. Gantes, Seismic risk assessment of buried pipelines at active fault crossings. Second European Conference on Earthquake Engineering and Seismology, Istanbul, Turkey, August 25-19, 2014.

[4] R.R. Youngs, W.J. Arabasz, R.E. Anderson, A.R. Ramelli, J.P. Ake, D.B. Slemmons, J.P. McCalpin, D.I. Doser, D.J. Fridrich, F.H. Swan III, A.M. Rogers, J.C. Yount, L.W. Anderson, K.D. Smith, R.LS. Bruhn, P.L.K. Knuepfer, R.B. Smith, C.M. dePolo, D.W. O’Leary, K.J. Coppersmith, S.K. Pezzopane, D.P. Schwartz, J.W. Whitney, S.S. Olig, G.R. Toro, A methodology for probabilistic fault displacements hazard analysis (PFDHA). Earthquake Spectra, 19(1), 191-219, 2003.

[5] K.L. Coppersmith, R.R. Youngs, Data needs for probabilistic fault displacement hazard analysis. Journal of Geodynamics, 29, 329-343, 2000.

[6] C.A. Cornell, Engineering seismic risk analysis. Bulletin of the Seismological Society of America. 58, 1583-1606, 1968.

[7] R. Gutenberg, C.F. Richter, Frequency of earthquakes in California. Bulletin of the Seismological Society of America, 34, 185-188, 1944.

[8] D.L. Wells, K.J. Coppersmith, New empirical relationships among magnitude, rupture length, rupture width, rupture area and surface displacement, Bulletin of Seismological Society of America, 84, 974-1002, 1994.

[9] P. Bazzuro, C.A. Cornell, Vector-valued probabilistic seismic hazard analysis (VPSHA). 7th U.S. National Conference on Earthquake Engineering, Boston, M.A., 2002.

[10] J.R. Benjamin, C.A. Cornell, Probability, Statistics and Decision for Civil Engineers. McGraw-Hill, New York, 1970.

[11] ALA American Lifelines Alliance, Guidelines for the Design of Buried Steel Pipe-July 2001 (with addenda through February 2005), 2001.

[12] J.J. Bommer, F. Scherbaum, The use and misuse of logic trees in probabilistic seismic hazard analysis. Earthquake Spectra, 24(4), 997-1009, 2008.

[13] European Committee for Standardization, Eurocode 8: Design of Structures for Earthquake Resistance, Part 4: Silos, Tanks and Pipelines. Brussels, 2004. 
[14] D.K. Karamitros, G.D. Bouckovalas, G.D. Kouretzis, Stress analysis of buried steel pipelines at strike-slip fault crossings. Soil Dynamics and Earthquake Engineering, 27, 200-211, 2007.

[15] D.K. Karamitros, G.D. Bouckovalas, G.D., Kouretzis, V. Gkesouli, An analytical method for strength verification of buried steel pipelines at normal fault crossings. Soil Dynamics and Earthquake Engineering, 31, 1452-1464, 2011. 